\title{
Synergistic effect of graphene nanoplatelets and carbon black in multifunctional EPDM nanocomposites
}

\author{
L. Valentini a, ${ }^{*}$, S. Bittolo Bon a , M.A. Lopez-Manchado ${ }^{b}$, R. Verdejo ${ }^{b}$, L. Pappalardo ${ }^{c}, A$ A. Bolognini ${ }^{d}, A$. \\ Alvino $^{e}$, S. Borsini e , A. Berardo f , N.M. Pugno f, g, h, ** \\ a Dipartimento di Ingegneria Civile e Ambientale, Universita di Perugia, UdR INSTM, Strada di Pentima 4, 05100 Terni, \\ Italy \\ b Instituto de Ciencia y Tecnología de Polímeros, ICTP-CSIC, Juan de la Cierva, 328006 Madrid, Spain \\ c Istituto Nazionale di Geofisica e Vulcanologia, Sezione di Napoli Osservatorio Vesuviano, via Diocleziano 328, I-80124 \\ Naples, Italy \\ a Dipartimento di Fisica e Laboratorio SERMS, Universita degli Studi di Perugia, Strada di Pentima 4, 05100 Terni, Italy \\ e SERMS srl, Strada di Pentima 8, 05100 Terni, Italy \\ ${ }^{f}$ Laboratory of Bio-Inspired and Graphene Nanomechanics, Department of Civil, Environmental and Mechanical \\ Engineering, University of Trento, Trento, Italy \\ $g$ Center for Materials and Microsystems, Fondazione Bruno Kessler, Trento, Italy \\ ${ }^{h}$ School of Engineering and Materials Science, Queen Mary University of London, Mile End Road, London, United \\ Kingdom
}

\begin{abstract}
Ethyleneepropyleneediene terpolymer rubber (EPDM)-based nanocomposites containing carbon black (CB), graphene nanoplatelets (GNPs), and mixtures of the two fillers were prepared. The influence of the relative amounts of the two fillers on the dynamic and static friction coefficients was examined. The static analysis of the coefficient of friction suggests that the partial substitution GNPs into the EPDM/CB blend did not produce a significant variation of the surface grip. The sample comprising EPDM/CB composite and an effective amount of GNPs dispersed in the matrix provides an increase of the thermal conductivity, damping (i. e. shock absorbing properties) and mechanical properties of the nanocomposites. The field-emission scanning electron microscopy and micro tomography analyses showed that the replacement of CB with GNPs reduces the CB aggregation and, hence, improving the percolation of the hybrid fillers and the interface resistance of the composite. The development of thermally conducting elastomeric nanocomposites could envisage their utilization in the processing of rubber blends satisfying the increasing demand to reduce both the duration of the vulcanization process and thus the cost of the vulcanized rubbers.
\end{abstract}

\section{Introduction}

Rubber is commonly considered the workhorse of the industrial and automotive products because of its good mechanical properties and its relatively low cost; finished products are found in the market place as compression molded products. The physical and chemical resistance properties of rubber materials are determined by the addition of carbon black (CB) that historically has been utilized to reinforce rubber matrices [1,2].

However, since the main factors that affect the composite properties are the particle size and the mode of interactions with the matrix materials, single filler does not generally match the structural and functional requirements of rubber advanced composites [3e7]. There are different types of carbon fillers (e. g. exfoliated graphite, carbon nanotubes and carbon fibres) and each type has its peculiar characteristic. For example, the percolation threshold of nanotubes is very low because of their high aspect ratio and it was found that the partial replacement of CB with carbon nanotubes 
leads to a much lower percolation threshold than that of the composite obtained with single filler and to a synergetic effect on the composite properties [8,9]; on the other hand the high cost hinders their large scale application. Exfoliated graphite is cheap and recently it was used as potential material for replacing $\mathrm{CB}$ for reinforcement of styrene-butadiene rubber. Its main drawback is the percolation threshold that is usually high and detrimental for the mechanical properties.

Thus, the use of a combination of different carbon fillers would be a good way to get balanced properties and cost. A model for predicting the synergy between electrical conducting nanofillers, assumed immiscible, was applied for the optimal design of real nanocomposites [10,11]. Ma et al. [12] showed that the addition of carbon nanotubes into CB polymer composites enhanced the electric conductivity of the polymer matrix [12] with a low percolation threshold of about $0.4 \mathrm{wt} \%$. The combination of two or more carbon fillers was demonstrated to improve also the thermal performance of the composite due to the synergistic effect $[13,14]$. Recently, Yang et al. [15] studied the effects of substituting $\mathrm{CB}$ with graphene oxide/CB and reduced graphene oxide/CB hybrid fillers on the structure and properties of natural rubber composites.

Carbon fillers with different aspect ratio and sizes can be mixed with a host polymer matrix showing evidences of exfoliation and shortening of the particle size during mixing process even with expanded graphite. That suggests that simple mechanical milling can facilitate the exfoliation of the graphite layers into smaller dimension. Das et al. [16] reported a TEM and X-ray diffraction study of styrene butadiene rubber composites showing that the dispersion/exfoliation of the stacked graphene sheets into individual single sheets was facilitated by the presence of carbon black in the system. The existence of few-layer graphene sheets was attributed to a complex morphology arisen from filler to filler network interaction. The same authors [17] reported another study on solution styrene butadiene rubber composites reinforced with graphene nanoplatelets, expanded graphite, and multiwalled carbon nanotubes. It was found that the high aspect ratio of carbon nanotubes enabled to form a network at low filler loading, leading to a good reinforcement effect.

Similarly to these previous attempts, Hu et al. [18] report a simple and effective way to disperse carbon nanotubes and graphene in silicone rubber and more recently Li et al. [19] demonstrated toughening natural rubber by designing a compact hybrid filler network composed of graphene and carbon nanotubes. In addition to graphene oxide, multi-layer graphene platelets also exhibit unique and useful behaviors. Multi-layer graphene, herein referred to as graphene nanoplatelets (GNPs) contains essentially no oxygen ( $<1 \%$ by weight of oxygen). GNPs are obtained from graphite expansion that determines the platelet thickness [20,21]. With this method, 2D graphite materials consisting of hundreds of stacked graphene layers with ABA or ABCA stacking, and with a thickness and/or lateral dimension less than $100 \mathrm{~nm}$ are obtained.

The 2D nanoscale dimension of GNPs is a huge benefit in relation to the large conventional 3D fillers [22]. Those graphitic inclusions are characterized by far better shape factor, larger contact surface and higher mechanical strength. At the same time GNPs tend to aggregate and are difficult to disperse in polymer matrices due to the strong van der Waals attraction between the sheets and their high surface area, the synergy among the hybrid fillers comprising of graphite intercalation compounds, mainly GNPs, and CB could lead to the development of graphite-based elastomer composites exhibiting exceptional mechanical and thermal properties.

It is known that rubbers or elastomers generally have a low thermal conductivity. Consequently, when such materials are used as packaging for electronic circuit, they store the generated heat that in turn raises the temperature of the device itself, thereby promoting heat deterioration of the electronic component. To achieve this goal, the heat conduction capability of a rubber may be improved by compounding a rubber with a filler having a heat conductivity higher than that of the rubber. 
High filler loadings (>30 vol\%) or traditional metallic materials were typically necessary to develop functional elastomers with appropriate level of thermal conductivity [23,24]. The employment of high filler loading makes difficult the processing, such as possibility to be extruded and injection molded, while traditional metallic materials with the highest thermal conductivity are too heavy and subjected to corrosion. Moreover the reinforcing capacity is deteriorated after some certain value of filler amount. When a dramatic increase in properties, such as mechanical and thermal properties can be achieved when two different fillers both with saturate amount are added in rubber matrix synchronously, this is suggestive of synergistic effect.

In this work a part of CB with GNPs was replaced to produce ethylene-propylene diene terpolymer rubber (EPDM) based nanocomposites; a proper combination of GNPs lead to synergistic effect in improving the thermal conductivity, damping and mechanical properties of the nanocomposites. The effects of substituting GNPs for CB on the thermal, damping and mechanical properties of rubber/CB composites was studied and rationalized in terms of a mixture model.

\section{Experimental details}

EPDM was kindly supplied by Exxon Mobil Chemical under the trade name Vistalon 7500 (ethylene content: $56.0 \mathrm{wt} \%$ and 5- ethylidene-2-norbornene (ENB) content: $5.7 \mathrm{wt} \%$ ). Carbon black was kindly supplied by Cabot, S.A. under the trade name Vulcan 3- N330 (diameter $225 \mathrm{~nm}$ with a surface area of $77 \mathrm{~m} 2 / \mathrm{g}$ ) and a paraffinic oil kindly supplied by Nynas, Nyflex 820 was used as plasticizer. GNPs, an intermediate grade between graphene and graphite, which can neither be considered pure graphene nor graphite were purchased from Cheap tubes. Rubber compounds were prepared in an open two-roll mill at room temperature. The rotors operated at a speed ratio of 1:1.4. The vulcanization ingredients were sequentially added to the elastomer before to the incorporation of the filler and sulphur. The recipes of the compounds are described in Table 1. Vulcanizing conditions (temperature and time) were previously determined by a Monsanto Moving Die Rheometer MDR 2000 E.

Table 1 Recipes of the rubber compounds (indicated in phr: parts per hundred of rubber). The \%weight content of GNPs/CB is reported below the name of each sample.

\begin{tabular}{|c|c|c|c|c|c|c|c|}
\hline Ingredient & EPDM-1 (0/0) & EPDM-2 (2/0) & $\operatorname{EPDM}-3(5 / 0)$ & EPDM-4 (10/0) & EPDM-5 $(0 / 48)$ & EPDM-6 $(2 / 24)$ & EPDM-7 (5/48) \\
\hline EPDM Vistalon 7500 & 100 & 100 & 100 & 100 & 100 & 100 & 100 \\
\hline Paraffinic oil & 80 & 80 & 80 & 80 & 80 & 80 & 80 \\
\hline Zinc oxide & 5 & 5 & 5 & 5 & 5 & 5 & 5 \\
\hline Stearic acid & 1 & 1 & 1 & 1 & 1 & 1 & 1 \\
\hline TMTD & 1.5 & 1.5 & 1.5 & 1.5 & 1.5 & 1.5 & 1.5 \\
\hline Sulphur & 3 & 3 & 3 & 3 & 3 & 3 & 3 \\
\hline Carbon black & - & - & - & - & 190 & 95 & 190 \\
\hline Graphene Nanoplatelets & - & 10 & 20 & 50 & - & 10 & 20 \\
\hline
\end{tabular}

Rubber compounds were then vulcanized at $160 \mathrm{C}$ in a thermofluid heated press. The vulcanization time of the samples corresponds to the optimum cure time t90 derived from the curing curves of the MDR 2000E. Specimens were mechanically cut out from the vulcanized plaques. Field emission scanning microscopy (FESEM) was used to investigate the cross section of the samples.

Tensile stress-strain properties were measured according to ISO 37e1977 specifications, on an Instron dynamometer (Model 4301), at $25 \mathrm{C}$ at a crosshead speed of $500 \mathrm{~mm} \min 1$. At least five specimens of each sample were tested.

XRD experiments were conducted with an XRD diffractometer (Bruker) with a radiation source of CuKa and wave length I $1 / 40.154 \mathrm{~nm}$ operating at $40 \mathrm{kV}$ and $40 \mathrm{~mA}$. The incidence angle (2q) was fixed between 1 and 60 and the scan rate was 0.02 /s. Raman measurements were performed with Labram Raman spectroscopy (Horiba, Jobin-Yvon spectrometer) with a wavelength of 632.8 $\mathrm{nm}$. 
A ball-on-disk tribometer was used to determine the dynamic friction coefficient of the composites. The samples were cut in order to have a squared base with different measures, from $8 \quad 8 \mathrm{~mm} 2$ to $1515 \mathrm{~mm} 2$ (average values), depending on the given materials. They were fixed in the tribometer and the antagonist material we chose was steel $(100 \mathrm{Cr} 6)$, a sphere of $6 \mathrm{~mm}$ diameter in order to have a single contact point between the rubber and the steel. No lubricants were used. The sliding velocity was set at $0.01 \mathrm{~m} / \mathrm{s}$ and the normal load varied from $0.05 \mathrm{~N}$ (softer samples) to $0.1 \mathrm{~N}$ (harder samples). For each sample from three to five measurements were realized.

The method used to measure the static friction coefficient is based on the Coulomb theory of friction. Each sample was positioned on a plate and fixed on it. After, a weight is put on the sample. The plate was then tilted until the stable configuration was overwhelmed and the weight slides on the rubber surface. The final configuration is tilted by a certain angle with respect to the initial position of the plate and corresponds to the transition from a stable state (static equilibrium) to an unstable one (incipient movement). The tangent of that angle corresponds to the ratio between the tangential force and the normal applied load (the weight). Five measures per sample were performed.

The damping properties were tested through a vibration generated via a pneumatic percussion system hitting a metallic plate. The impact area is a metallic plate where the sample to be tested has been fastened to. The sample was hit by a percussion which excites the vibration. A shock accelerometer positioned in the back plate is thus excited and the response is recorded and digitalized via high performance data acquisition system. The impact velocity was set to $8 \mathrm{~m} / \mathrm{s}$ resulting in an impact energy of $58 \mathrm{~J}$. Three tests were repeated on each sample; the experimental error was estimated below $1 \%$.

Thermal conductivity measurements follow the "two thermometer-one heater" method using a custom built stage. Two PT100 thermocouples, contacted to the surfaces of a $13 \quad 40 \mathrm{~mm} 2$ rectangular shape and $14 \mathrm{~mm}$ thick sample, monitor the temperature of two polished oxygen-free sample sides. A $3.4 \mathrm{Ohm}$ resistor heats the top plate (13 $40 \mathrm{~mm} 2$ surface, $14 \mathrm{~mm}$ thick) to a temperature THot. Heat flows from the top plate, through the sample, and into the bottom plate which is thermally grounded to TCold (i. e. 20 C) by the cold plate. Thermally conducting grease was used to enhance the thermal contact to the bottom of the sample.

The microstructure of the sample was investigated by micro tomography (m-CT) using a Carl Zeiss Xradia Versa-410 3D X-ray microscope. The scan was performed over a 360 rotation using 1600 projections, $80 \mathrm{KV}$ voltage, $7 \mathrm{~W}$ power, $80 \mathrm{~s}$ exposure time, and 20 objective lens. The resulting nominal voxel (volumetric pixel) size was $0.36 \mathrm{~mm}$ and the total scan time was $\sim 38 \mathrm{~h}$ for each scan. Reconstruction of the attenuation data was performed using filtered back-projection, producing a stack of 967 cross-sectional, grey-scale digital images. The different components and their distributions have been analysed by segmenting regions of a given range of grayscale values from the rest of the image by using the XM3D viewer and Fiji software.

\section{Results and discussion}

Raman spectroscopy has been carried out to elucidate the Raman characteristics of GNP used in this work, such as differentiating few-layer and multi-layer from bulk graphite [25] and detecting structural defects [26]. The main features in Raman spectra reported in Fig. 1a are the $G$ and $D$ bands and the second order of the $D$ band, so-called 2D band. The $G$ band, standing at around 1580 $\mathrm{cm} 1$, corresponds to in-plane carbon-atom stretching vibrations [26]. The positions of the $D$ and 2D bands are excitationenergy dependent and occur at around $1329 \mathrm{~cm} 1$ and $2663 \mathrm{~cm} 1$, respectively (Fig. 1a). The $D$ band is activated by the presence of defects [26]. After characterizations of a large number of ABstacked few-layer graphene samples during the past years [27], the FWHM of 2D band (Fig. 1b) unambiguously is associated to the graphite layer number. The typical FWHM of 2D peaks 
plotted in Fig. 1b is consistent with five-layer graphitic material and thus with GNPs with AB stacking, and having a thickness and/or lateral dimension less than $100 \mathrm{~nm}$ [27].

GNPs have attracted considerable attention in nanocomposites, thanks to the excellent in-plane mechanical and thermal properties of graphene. The stress-strain characteristics of the prepared nanocomposites are presented in Fig. $2 \mathrm{a}$ and the tensile properties given in terms of the modulus at different strains (50\%, 100\% and 300\%), maximum strength and elongation at break are reported in Figs. $2 \mathrm{~b}$ and 3 , respectively. It is known that $\mathrm{CB}$ or silica when added to elastomers create a modulus that increases with strain. This non-linearity protects rubber from damage during large deformations [28]. Pristine GNPs provide enhanced non-linear strength to elastomers. The interface is similar to that of $\mathrm{CB}$, the flexibility of the GNPs enables deformation at low strains and strengthening at higher deformations. As expected, the addition of the fillers to the EPDM matrix gives rise to an increase of the stiffness of the material which is reflected in an improvement of the modulus at different strains (Fig. 2). The elongation at break, as an indicator for the toughness of the materials, decreases when adding GNPs to the EPDM/CB blend (Fig. 3) (i. e. EPDM-7 sample). The synergistic effect of CB and GNPs is evident in the sample EPDM-6 (i. e. 2 wt $\%$ of GNPs and 24 wt $\%$ of CB) that showed a higher increment of the maximum strength (Fig. 2) along with a higher elongation at break with respect to the EPDM/CB blends (Fig. 3).
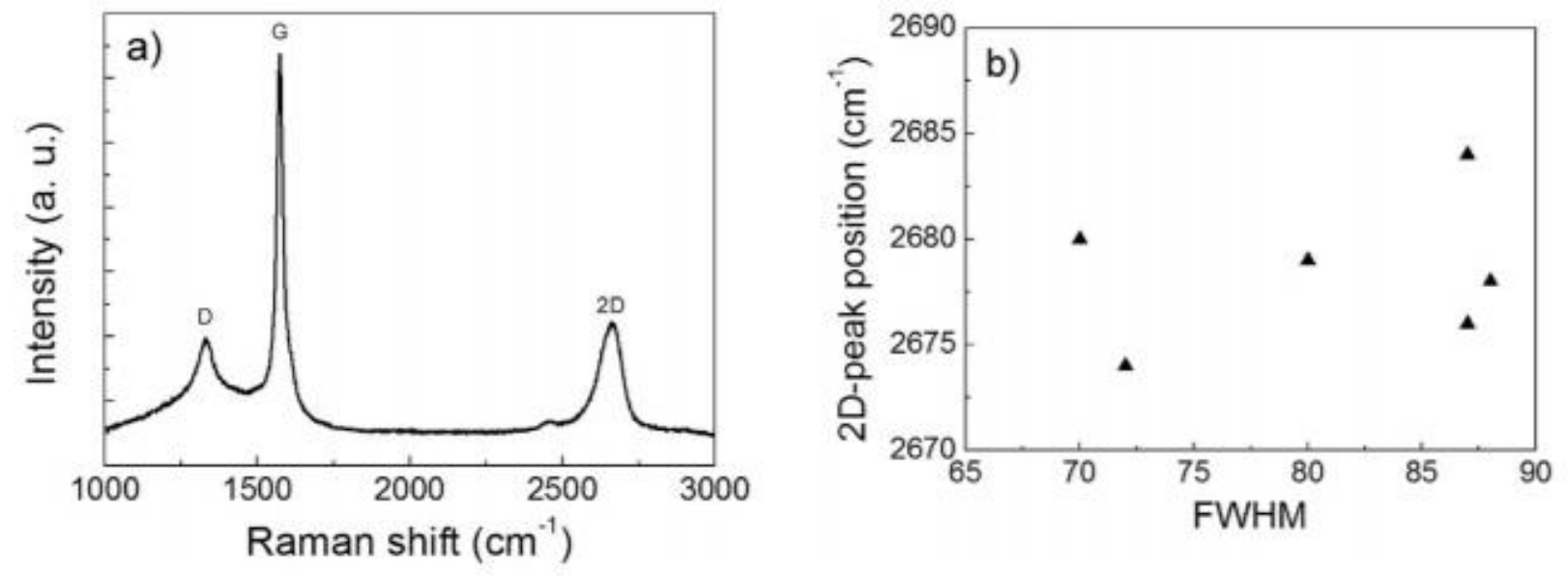

Fig. 1. a) Raman spectra of GNPs. b) The data of FWHM with respect to 2D peak positions.

For a system containing two types of fillers such as GNPs and CB, our results can be rationalized in terms of the following mixture model:

$$
\sigma=\sigma_{\mathrm{m}}\left(1-\mathrm{f}_{\mathrm{GNPs}}-\mathrm{f}_{\mathrm{CB}}\right)+\sigma_{\mathrm{GNPs}} \mathrm{f}_{\mathrm{GNPs}}+\sigma_{\mathrm{CB}} \mathrm{f}_{\mathrm{CB}}
$$

where $s$ is the composite strength, sm is the matrix strength, sGNPs is the GNP strength, $s C B$ is the CB strength and fGNPs and $\mathrm{FCB}$ are the GNPs and CB concentrations, respectively.

Assuming sGNPs $=800 \mathrm{MPa}$ [29], sCB $\sim 14 \mathrm{MPa}$ (obtained by using the rule of mixture for $\mathrm{CB}$ single phase $s$ y sm(1 0.48)p0.48sCB) and fGNPs and fCB the concentrations for obtaining the maximum of the mechanical resistance, the model predict for the composite a mechanical strength of about 25 $\mathrm{MPa}$ that is in good agreement with that obtained experimentally (i. e. 20 MPa). For a single phase inclusion, if an ideal dispersion, thus without agglomeration, is considered, the composite mechanical resistance would depend linearly on the percentage of the phase itself, thus an increment of $f$ would cause an increment also in the mechanical resistance (assuming the strength of the inclusion much larger than that of the matrix). This is not true if the agglomeration of the phase takes place. In the model for two immiscible phases, the synergy can be understood as a retardation of the agglomeration towards higher total concentration as reported below. 

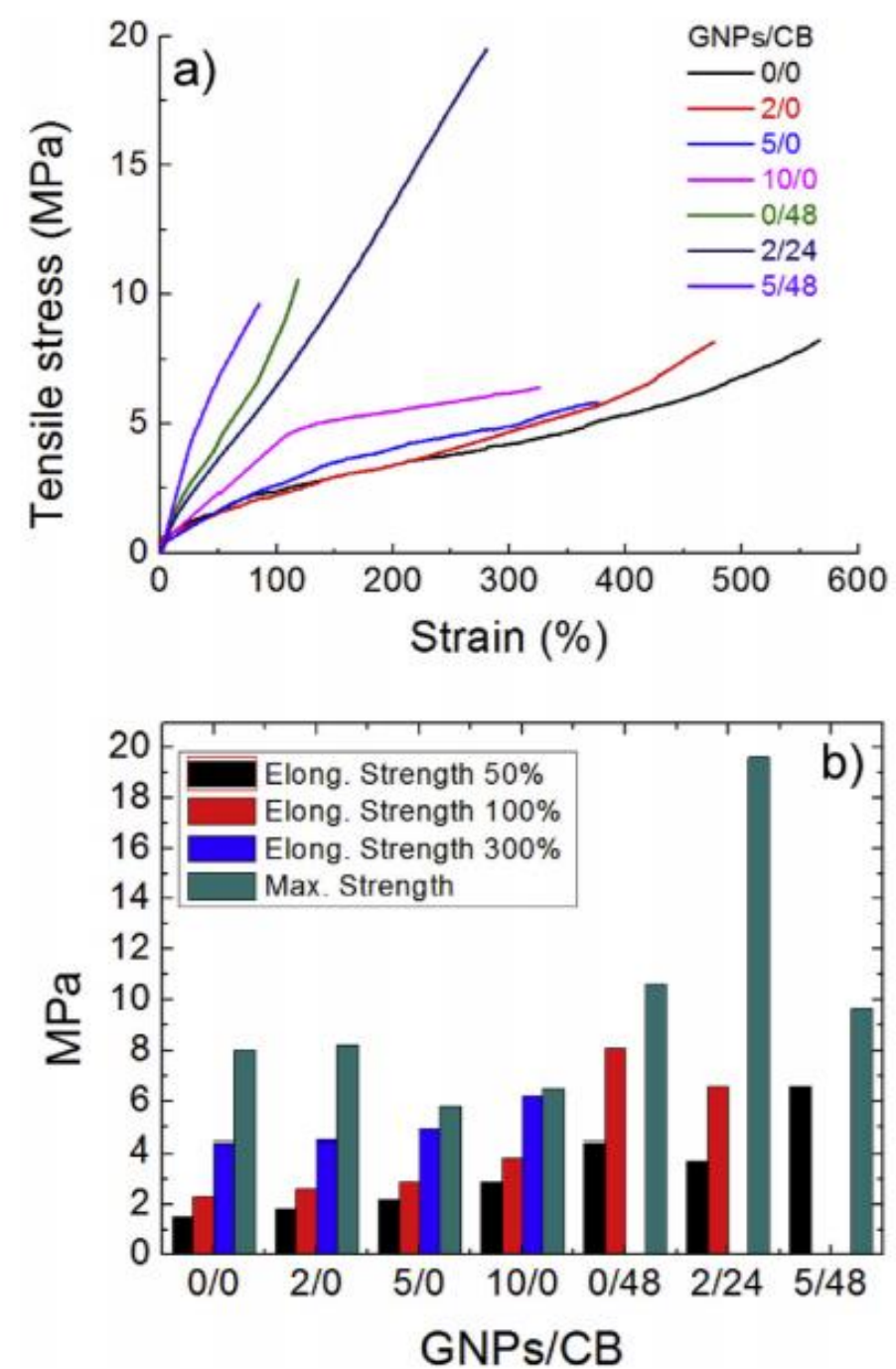

Fig. 2. (a) Stress-strain curves of the prepared samples. (b) Modulus at different strains and maximum strength of the prepared samples.

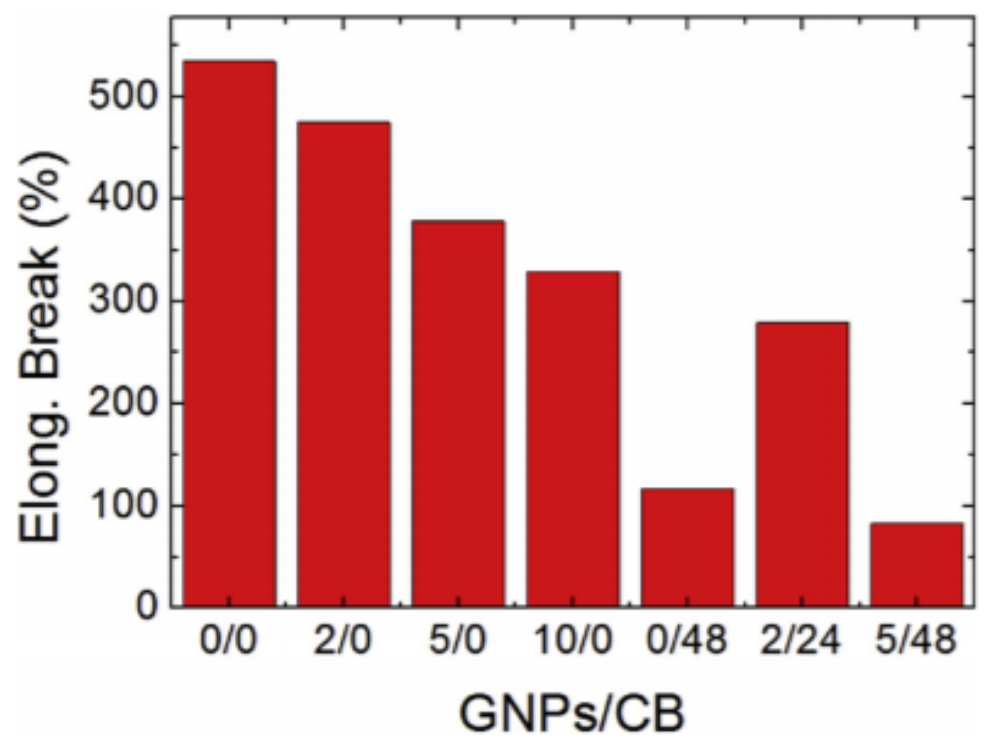

Fig. 3. Elongation at break of the prepared samples. 


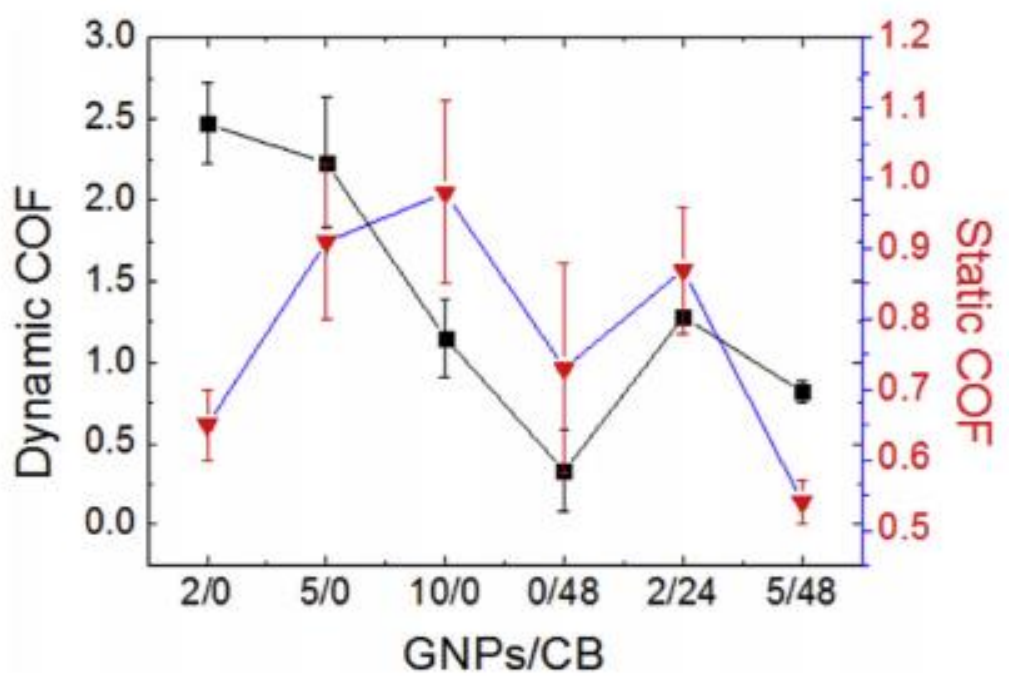

Fig. 4. Dynamic and static coefficient of friction measured of the prepared samples.
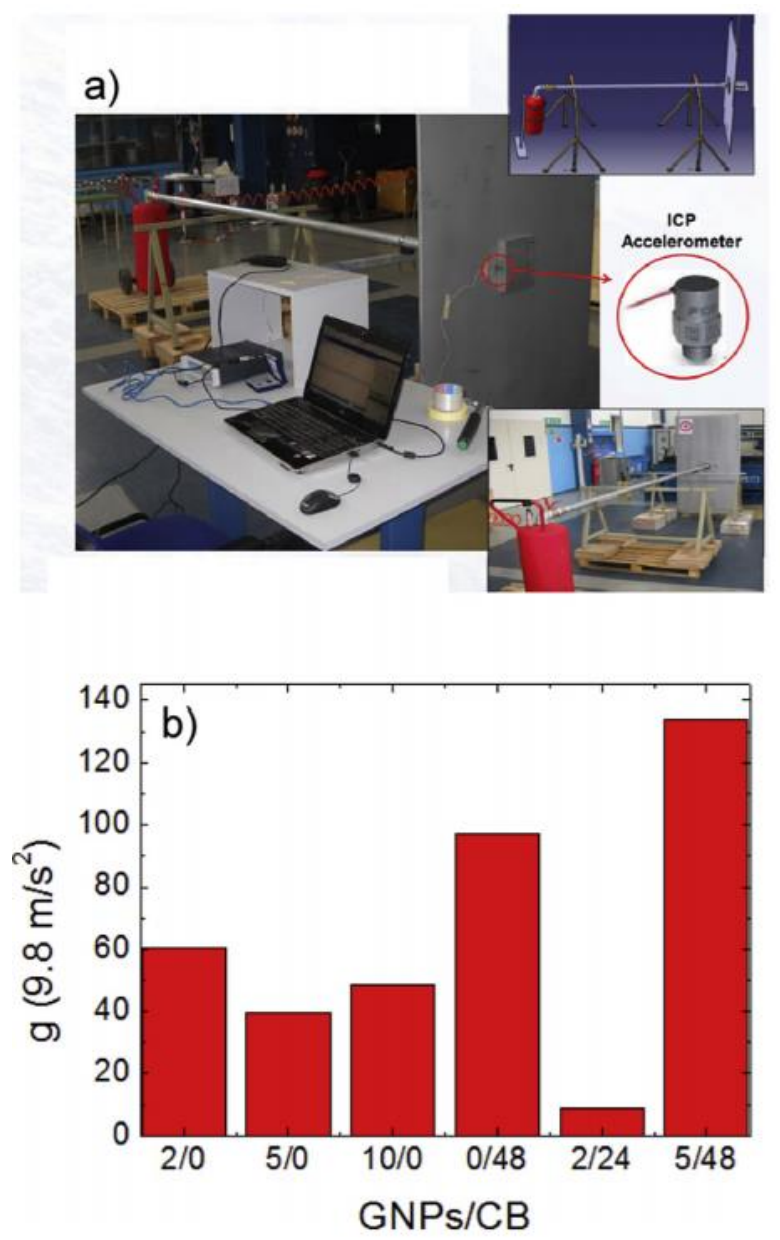

Fig. 5. a) Set-up of the impact test. The impact area is a metallic plate where the sample to be tested has been fastened to. The sample was hit by a percussion which excites the vibration. A shock accelerometer positioned in the back plate is thus excited and the response is recorded and digitalized via high performance data acquisition system. b) Peak acceleration measured by the accelerometer in the impact test of the prepared samples.

The dynamic friction coefficients of the samples were estimated accordingly to the Hertzian analysis for a smooth sphere in contact with a smooth flat surface, where the radius of contact circle expressed as a $1 / 4[3 L R / 4 E]^{\wedge} 1 / 3$, where $L$ is the applied load, $R$ is the sphere radius and $E$ is the elastic modulus of the softer material (i. e. rubber). In the present case the only parameter varied was the load, thus accordingly to the mechanical properties, it was decreased for the softer 
composite samples containing a GNP/CB ratio of $2 / 0,5 / 0$ and $10 / 0$, respectively. The final values are shown in Fig. 4. For composites with a GNP/CB ratio of 5/0, 10/0 and 2/24 values major than 1 were obtained and in literature for particular combinations of rubbers similar results were found (i. e. rubber-steel contact) [30e34]. It was also reported that the dynamic friction coefficient depends on the sliding velocity, it increases if the velocity increases, but becomes almost stable for velocities from $0.01 \mathrm{~m} / \mathrm{s}$ and more [30e34].

The static coefficient of friction of the samples was estimated by putting a weight made of steel ( 0.7 g) on the rubber samples and tilting the plate, until the incipient sliding was reached. The dynamic and static coefficients of friction are not comparable due to the different type of steel used as counterpart as well as the different type of setup adopted for dynamic and static tests. The addition of GNPs to the EPDM/CB blend reduces the static coeffi- cient of friction while the partial substitution of CB with GNPs did not affect the grip of the EPDM/CB sample surfaces.

Fig. 5 reports the peak acceleration measured in the impact excitation test. The damping of the sample can be qualitatively estimated by the peak acceleration. No data were recorded on neat EPDM due to the cracking of the samples even at lower impact velocity. It is evident how in the composites, the impact performances depend on the elongation at break; in particular, the higher stiffness of the composites with CB shows a scarce damping properties. The obtained results can be explained with the increase of the modulus at different strains along with the reduction of elongation when the GNPs were added. The addition of $5 \mathrm{wt} \%$ of GNPs in the $48 \mathrm{wt} \% \mathrm{CB}$ filled matrix deteriorates the damping properties. The partial substitution of CB in the sample with $2 \mathrm{wt} \%$ of GNPs and 24 wt\% of CB showed the best shock absorbing performance with a lower variation of the acceleration peak after the impact.

Fig. 6a shows the experimental set up for the thermal conductivity measurements. The in-plane thermal conductivity was measured through a rectangular shaped $(1.5 \mathrm{~cm}$ wide, $1.4 \mathrm{~mm}$ high, $4 \mathrm{~cm}$ long) specimen, as shown in Fig. 5a. The in-plane thermal conductivity test method was used with the goal to conduct heat only by conduction through the solid sample. A Mylar cap around the cold plate fixed at TCold and a high vacuum 105 Torr reduce thermal losses due to radiation and convection, respectively. When the sample is powered, the generated heat flows through the sample from the sample heater to the cold plate. Heat is generated in the sample from electrical resistance heating of the sample heater. Thus, heat is equal to the power dissipated by the resistor (V I). Fig. $6 a$ illustrates this set up. The thermal conductivity of the specimen is determined by Ref. [35] 11/4(V

I/DT) (d/A) where I is the thermal conductivity of the specimen being tested; $V$ is the voltage drop across the sample heater resistor; I is the current through the sample heater resistor; DT is the temperature difference across the specimen; $d$ is the distance between the two junctions of the thermocouples; and $A$ is the cross-sectional area of the specimen (specimen width specimen height). Such in-plane thermal conductivity test method is based on the steady state method (see inset of Fig. 6a).

In analogy with electrical conductivity, the thermal conductivity of polymers filled with conducting nanoparticles derives from the formation of a percolation network of the fillers in the matrix [36]. The increasing of the conductive paths enhances the composite thermal conductivity. As for the CB filler alone EPDM-5 (i. e. 48 wt\% CB), the conductive network is formed due to the contact between GNPs and CB. For the sample EPDM-6 (i. e. 2 wt\% GNPs and 24 wt $\%$ CB) when GNPs are added into the CB composite, GNP particles act as spacers between the CB agglomerates, which leads to the formation of linked conductive paths (Fig. 6b). As proof of this statement, XRD, FESEM and MCT analyses were performed.

XRD experiments on GNPs and CB related rubber composites are reported in Fig. 7a. All XRD spectra present a broad region at $2 q^{1 / 4} 14$ e20 due to the EPDM polymer reported in Fig. 7b [37]. 


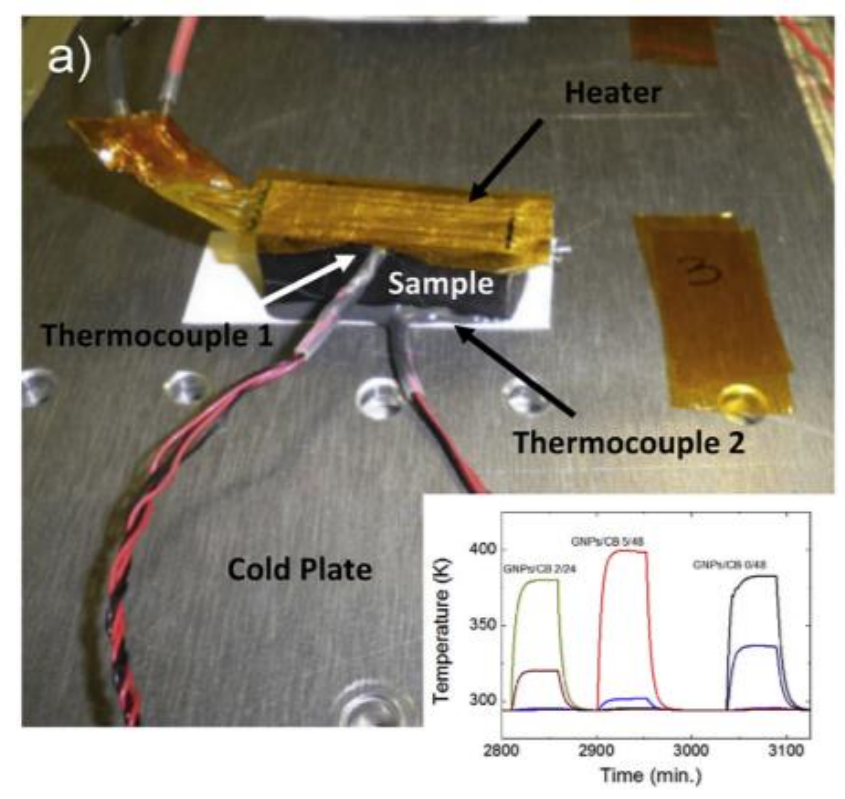

b)

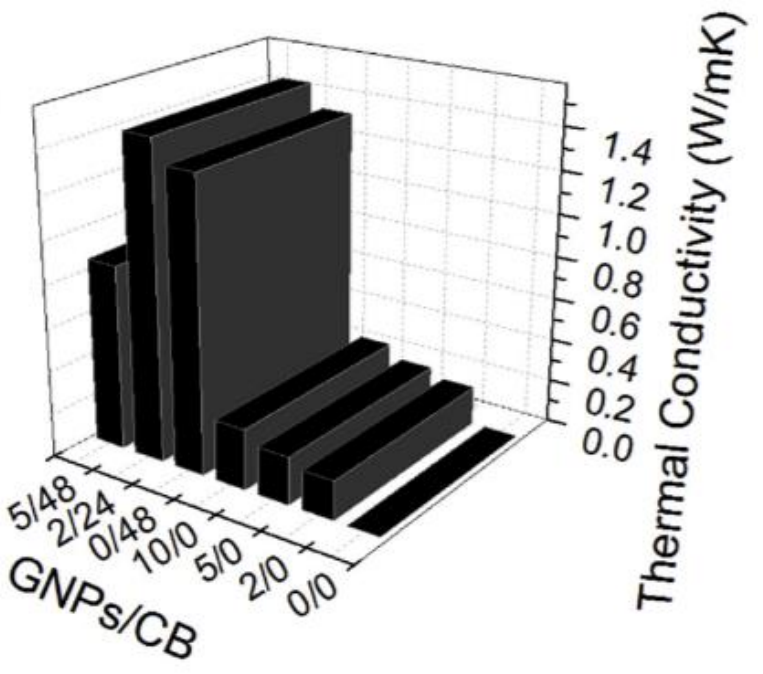

Fig. 6. (a) Set up of the thermal conductivity measurements. (b) Thermal conductivity values as a function of the GNPs/CB content.

Fig. 7b shows the X-ray diffractogram recorded for the pristine carbon black; the spectrum reveals a peak at about $2 q^{1 / 4} 24.6$, which is the $d(002) 3.72 \AA$ lattice spacing of the graphite layers [38,39]. XRD pattern of the EPDM-5 (i. e. 48 wt\% CB) with CB filler alone, reveals that the EPDM region is followed by a distinct crystalline region at $2 q 1 / 426.5$. This results is in agreement with a previous study reported in Ref. [40], stating that carbon black aggregates tend to concentrate in amorphous regions of the polymer matrix; the peak at $2 q$ of about 32 corresponds to (100) lattice plane of the hexagonal wurtzite structure of zinc oxide $(\mathrm{ZnO})$ [41].

XRD pattern of EPDM-3 (i. e. 5 wt\% GNPs) shows three signifi- cant peaks at $2 q$ of about 32 , 34 and 36 corresponding to (100), (001) and (101) lattice planes of the hexagonal wurtzite structure of zinc oxide ( $\mathrm{ZnO})$, respectively [41]. By comparison with the XRD pattern of pristine GNPs reported in Fig. 7b, it is evident that the peak at about 26.3 is due to the GNPs. The diffraction peak at about 11.7 is attributed to the intrinsic diffraction of oxidized graphite, as confirmed by the XRD pattern of oxidized graphite reported elsewhere [42] and indicated by Raman measurement that there are substantial defects on graphite plane prone to be oxidized during the vulcanization process. 

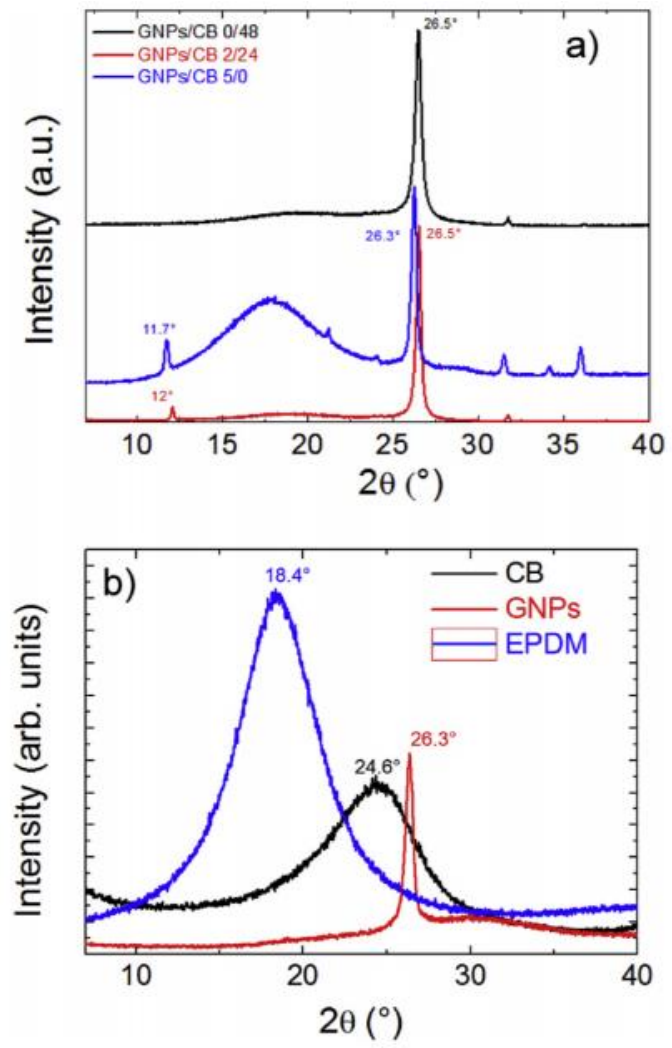

Fig. 7. X-ray diffraction patterns of (a) EPDM nanocomposites with different GNPs/CB content and (b) $\mathrm{CB}, \mathrm{GNPs}$ and neat EPDM.
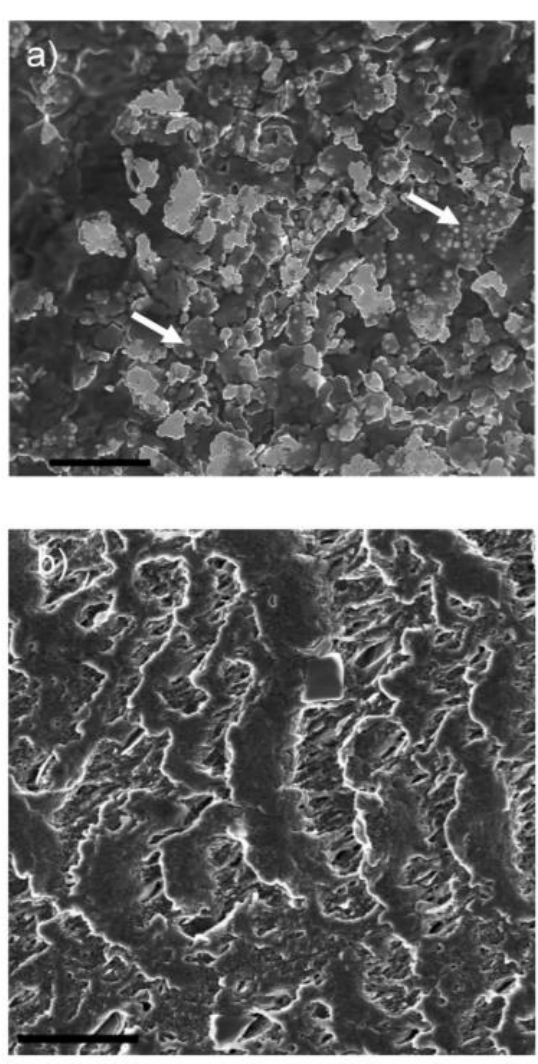

Fig. 8. FESEM images of the a) EPDM- 6 and b) EPDM-7 samples. The arrows in Fig. 6a) shows the $\mathrm{CB}$ agglomerates on a GNP sheet. The scale bars indicate 1 $\mathrm{mm}$.

Accordingly to Wei et al. [36], our FESEM analysis reported in Fig. 8a shows small CB agglomerates attached on the surface and edge of the GNPs. On the other hand, increasing the GNPs content into the sample with the highest CB concentration (i. e. $5 \mathrm{wt} \%$ GNPs and $48 \mathrm{wt} \% \mathrm{CB}$ ) contribute to the increase of the $\mathrm{CB}$ agglomeration resulting in a decrease of both impact properties and thermal conductivity (Figs. 6b and 8b).

Fig. 9 shows the results of m-CT analyses for the sample EPDM-6 (i. e. 2 wt\% GNPs and 24 wt\% $\mathrm{CB}$ ). The raw data acquired by the X-ray microscope have been processed using tomographic reconstruction, producing a stack of 967 cross-sectional, grey-scale digital images. The total cylindrical volume of the analysed sample has $348 \mathrm{~mm}$ in diameter and $360 \mathrm{~mm}$ in height (Fig. 9a). As the grey level value in 3D image is related to the $X$-ray absorption of the material, the concentrated CB agglomerates are shown in lighter grey to white color, while GNPs due to its flat shape is shown in darker grey color.

Image analysis techniques have been applied in order to segment the 3D imaged data and separate the different materials by their grey level value. Segmented $\mathrm{m}-\mathrm{CT}$ images reported in Fig. $9 \mathrm{~b}$ and c, show that both GNPs and CB are uniformly dispersed within the polymer matrix. Particularly CB forms aggregates (Fig. 9d) mostly on the surface of the GNPs linking the gap distance between the GNPs resulting in the formation of additional conductive paths and increasing the interface resistance in the hybrid composite. 


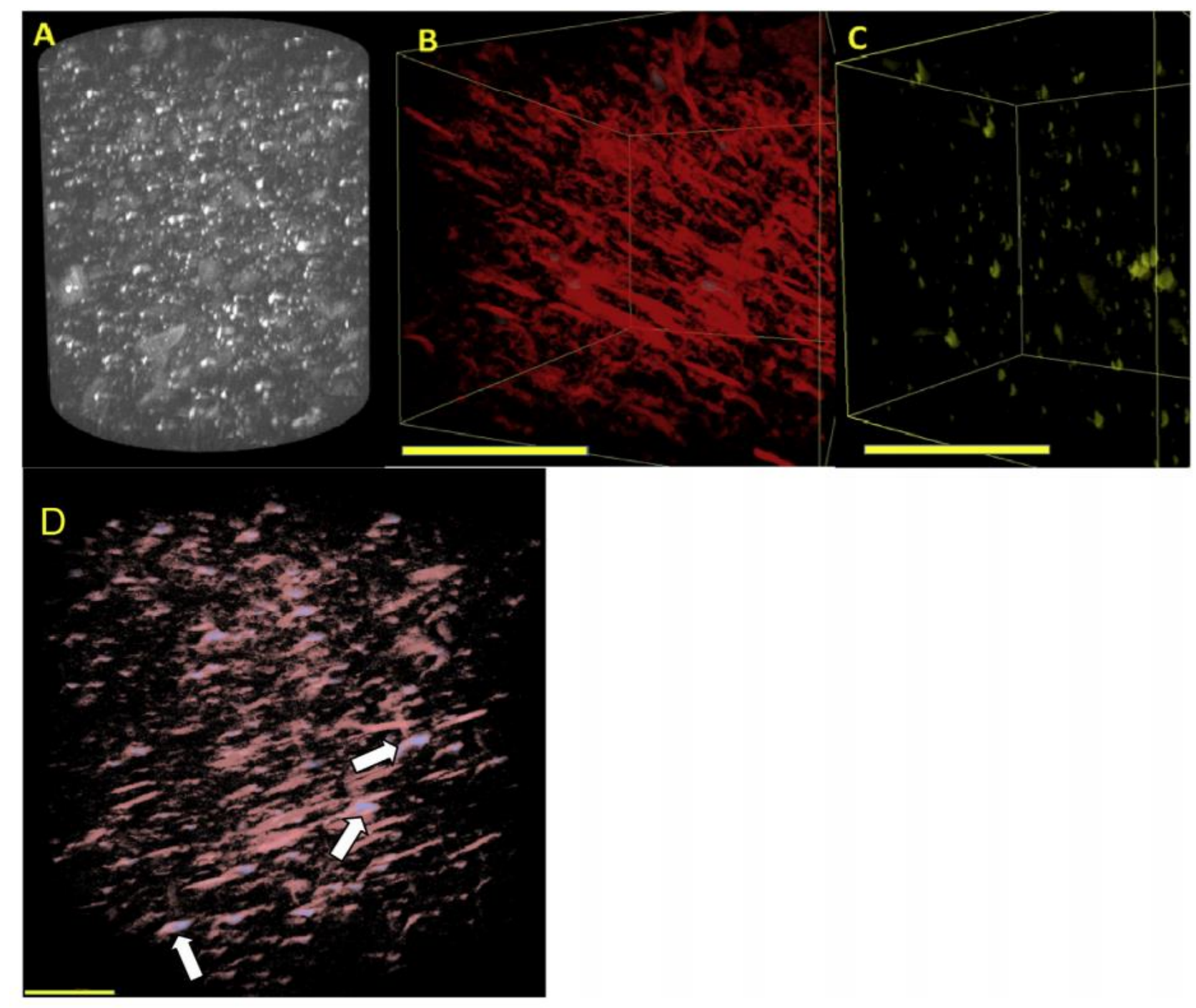

Fig. 9. A) Total cylindrical volume of the EPDM-6 sample (348 mm in diameter and $360 \mathrm{~mm}$ in height); B) and C) Detail of segmented $\mathrm{m}-\mathrm{CT}$ images showing the distribution of the GNPs and CB aggregates, respectively (scale bar is $50 \mathrm{~mm}$ ). D) Detail of segmented $\mathrm{m}-\mathrm{CT}$ images showing the whole distribution of the GNPs (in red) and CB aggregates (in blue indicated by the arrows) (scale bar is $50 \mathrm{~mm}$ ).

\section{Conclusions}

In this paper we adopt a processing technology to develop elastomer plus nano-graphite hybrid composites with multifunctional properties. Beyond the improvements of the mechanical properties, the research findings demonstrate the synergistic effect of carbon black and graphene nanoplatelets to prepare rubber composites thermally conductive and to design a new class of shock absorbers. It was found that a critical GNPs/CB ratio was able to reduce the strong interlayer forces among the GNPs sheets, which led to the efficiency on reinforcement in mechanical properties and improvements of the performance of the rubber composites.

\section{Acknowledgements}

NMP is supported by the European Research Council (ERC StG Ideas 2011 BIHSNAM n. 279985 on "Bio-Inspired hierarchical supernanomaterials", ERC PoC 2013 KNOTOUGH n. 632277 on "Supertough knotted fibers", ERC PoC 2015 SILKENE nr. 693670 on "Bionic silk with graphene or 
other nanomaterials spun by silkworms"), by the European Commission under the Graphene Flagship (WP10 "Nanocomposites", n. 604391) and by the Provincia Autonoma di Trento ("Graphene Nanocomposites", n. S116/2012- 242637 and delib. reg. n. 2266). The authors thank MINECO for the partial financial support of this work (project MAT2013-48107-C3). Dr. Manoj Tripathi (Center for Materials and Microsystems, Fondazione Bruno Kessler, Trento e Italy) is gratefully acknowledged for Raman measurements.

\section{References}

[1] N. Rattanasom, T. Saowapark, C. Deeprasertkul, Reinforcement of natural rubber with silica/carbon black hybrid filler, Polym. Test. 26 (2007) 369e377.

[2] Yimin Zhang, S. Ge, B. Tang, T. Koga, M.H. Rafailovich, J.C. Sokolov, D.G. Peiffer, Z. Li, A.J. Dias, K.O. McElrath, M.Y. Lin, S.K. Satija, S.G. Urquhart, H. Ade, D. Nguyen, Effect of carbon black and silica fillers in elastomer blends, Macromolecules 34 (2001) $7056 e 7065$.

[3] M.J. Wang, K. Mahmud, L.J. Murphy, W.J. Patterson, Kautsch. Gummi Kunstst. 51 (1998) 348.

[4] L.L. Qu, G.S. Huang, P. Zhang, Y.J. Nie, G.S. Weng, J.R. Wu, Synergistic reinforcement of nanoclay and carbon black in natural rubber, Polym. Int. 59 (2010) 1397e1402.

[5] R. Verdejo, M.A. Lopez-Manchado, L. Valentini, J.M. Kenny, Carbon nanotube reinforced rubber composites, in: S. Thomas, R. Stephen (Eds.), Rubber Nanocomposites, Wiley, Singapore, 2010, pp. 147e168. Chapter 6.

[6] Z. Wang, X.Y. Meng, J.Z. Li, X.H. Du, S.Y. Li, Z.W. Jiang, T.A. Tang, Simple method for preparing carbon nanotubes/clay hybrids in water, J. Phys. Chem. C 113 (2009) 8058e8064.

[7] Z.H. Tang, Q.Y. Wei, T.F. Lin, B.C. Guo, D.M. Jia, The use of a hybrid consisting of tubular clay and graphene as a reinforcement for elastomers, RSC Adv. 3 (2013) 17057e17064.

[8] S.M. Zhang, L. Lin, H. Deng, X. Gao, E. Bilotti, T. Peijs, Q. Zhang, Q. Fu, Synergistic effect in conductive networks constructed with carbon nanofillers in different dimensions, Express Polym. Lett. 6 (2012) 159e168.

[9] M. Galimberti, M. Coombs, P. Riccio, T. Ricco, S. Passera, S. Pandini, L. Conzatti, A. Ravasio, I. Tritto, The role of CNTs in promoting hybrid filler networking and synergism with carbon black in the mechanical behavior of filled polyisoprene, Macromol. Mater. Eng. 298 (2013) 241e251.

[10] Y. Mao, S. Wen, Y. Chen, F. Zhang, P. Panine, T.W. Chan, L. Zhang, Y. Liang, L. Liu, High performance graphene oxide based rubber composites, Sci. Rep. 3 (2013) 2508e2515.

[11] Y. Sun, H.-D. Bao, Z.-X. Guo, J. Yu, Modeling of the electrical percolation of mixed carbon fillers in polymer-based composites, Macromolecules 42 (2009) $459 \mathrm{e} 463$.

[12] P.C. Ma, M.Y. Liu, H. Zhang, S.Q. Wang, R. Wang, K. Wang, Enhanced electrical conductivity of nanocomposites containing hybrid fillers of carbon nanotubes and carbon black, Acs Appl. Mater. Interfaces 1 (2009) 1090e1096.

[13] E.H. Weber, M.L. Clingerman, J.A. King, Thermally conductive nylon 6,6 and polycarbonate based resins. I. Synergistic effects of carbon fillers, J. Appl. Polym. Sci. 88 (2003) 112e122.

[14] G.W. Lee, M. Park, J. Kim, J.I. Lee, H.G. Yoon, Enhanced thermal conductivity of polymer composites filled with hybrid filler, Compos. A 37 (2006) $727 e 734$. 
[15] G. Yang, Z. Liao, Z. Yang, Z. Tang, B. Guo, Effects of substitution for carbon black with graphene oxide or graphene on the morphology and performance of natural rubber/carbon black composites, J. Appl. Polym. Sci. 132 (2015) 41832.

[16] A. Das, R. Boldt, R. Jurk, D. Jehnichen, D. Fischer, R. Werner Stockelhuber, G. Heinrich, Nanoscale morphological analysis of graphene erubber composites using 3D transmission electron microscopy, RSC Adv. 4 (2014) 9300e9307.

[17] A. Das, R. Boldt, R. Jurk, D. Jehnichen, D. Fischer, R. Werner Stockelhuber, G. Heinrich, Rubber composites based on graphene nanoplatelets, expanded graphite, carbon nanotubes and their combination: a comparative study, Comp. Sci. Technol. 72 (2012) 1961e1967.

[18] H. Hu, L. Zhao, J. Liu, Y. Liu, J. Cheng, J. Luo, Y. Liang, Y. Tao, X. Wang, J. Zhao, Enhanced dispersion of carbon nanotube in silicone rubber assisted by grapheme, Polymer 53 (2012) $3378 \mathrm{e} 3385$.

[19] H. Li, L. Yang, G. Weng, W. Xing, J. Wu, G. Huang, Toughening rubbers with a hybrid filler network of graphene and carbon nanotubes, J. Mater. Chem. A 3 (2015) 22385 e22392.

[20] D.D.L. Chung, Exfoliation of graphite, J. Mater. Sci. 22 (1987) 4190e4198.

[21] D.D.L. Chung, Review graphite, J. Mater. Sci. 37 (8) (2002) 1475e1489.

[22] N. Pugno, Nanotube Superfiber Materials: Changing Engineering Design, 2013, pp. 495e518.

[23] S. Araby, I. Zaman, Q. Meng, N. Kawashima, A. Michelmore, H.-C. Kuan, P. Majewski, J. Ma, L. Zhang, Melt compounding with graphene to develop functional, high-performance elastomers, Nanotechnology 24 (2013) 165601.

[24] Z. Han, A. Fina, Thermal conductivity of carbon nanotubes and their polymer nanocomposites: a review, Prog. Polym. Sci. 36 (2011) 914e944.

[25] Editorial All in the graphene family e a recommended nomenclature for twodimensional carbon materials, Carbon 65 (2013) 1 e6.

[26] A.C. Ferrari, J.C. Meyer, V. Scardaci, C. Casiraghi, M. Lazzeri, F. Mauri, S. Piscanec, D. Jiang, K.S. Novoselov, S. Roth, A.K. Geim, Raman spectrum of graphene and graphene layers, Phys. Rev. Lett. 97 (2006) 187401.

[27] Y. Hao, Y. Wang, L. Wang, Z. Ni, Z. Wang, R. Wang, C.K. Koo, Z. Shen, J.T.L. Thong, Probing layer number and stacking order of few-layer graphene by Raman spectroscopy, Small 6 (2010) $195 \mathrm{e} 200$.

[28] M. Tosaka, D. Kawakami, K. Senoo, S. Kohjiya, Y. Ikeda, S. Toki, B.S. Hsiao, Crystallization and stress relaxation in highly stretched samples of natural rubber and its synthetic analogue, Macromolecules 39 (2006) 5100e5105.

[29] L. Valentini, S. Bittolo Bon, S. Signetti, N.M. Pugno, Graphene based bionic composites with multifunctional and repairing properties, ACS Appl. Mater. Interfaces (2016), http://dx.doi.org/10.1021/acsami.6b02530.

[30] F.L. Roth, R.L. Driscoll, W.L. Holt, Frictional properties of rubber, Rubber Chem. Technol. 16 (1943) $155 \mathrm{e} 177$.

[31] W. Leilei, L. Zhang, M. Tian, Effect of expanded graphite (EG) dispersion on the mechanical and tribological properties of nitrile rubber/EG composites, Wear 276 (2012) 85e93.

[32] S.H. Song, H.K. Jeong, Y.G. Kang, Preparation and characterization of exfoliated graphite and its styrene butadiene rubber nanocomposites, J. Ind. Eng. Chem. 16 (2010) 1059e1065. 
[33] J. Karger-Kocsis, A. Mousa, Z. Major, N. Bek esi, Dry friction and sliding wear of EPDM rubbers against steel as a function of carbon black content, Wear 264 (2008) 359e367.

[34] D. Felhos, J. Karger-Kocsis, Tribological testing of peroxide-cured EPDM rub- $€$ bers with different carbon black contents under dry sliding conditions against steel, Tribol. Int. 41 (2008) $404 \mathrm{e} 415$.

[35] A. Demain, J.P. Issi, Effective thermal conductivity of composites with interfacial thermal barrier resistance, J. Compos. Mater. 27 (1993) 668e683.

[36] T. Wei, L. Song, C. Zheng, K. Wang, J. Yan, B. Shao, Z.-J. Fan, The synergy of a three filler combination in the conductivity of epoxy composites, Mater. Lett. 64 (2010) 2376e2379.

[37] X. Hu, H. Gao, X. Zhou, Y. Cui, H. Ge, A new approach to rubber reinforcement, RSC Adv. 4 (2014) 13662e13668.

[38] F. Charreteur, F. Jaouen, S. Ruggeri, J.-P. Dodelet, Fe/N/C non-precious catalysts for PEM fuel cells: influence of the structural parameters of pristine commercial carbon blacks on their activity for oxygen reduction, Electrochim. Acta 53 (2008) 2925e2938.

[39] J. Biscoe, B.E. Warren, An X-ray study of carbon black, J. Appl. Phys. 13 (1942) 364e371.

[40] S.N. Lawandy, S.F. Halim, N.A. Darwish, Structure aggregation of carbon black in ethylenepropylene diene polymer, eXPRESS Polym. Lett. 3 (2009) $152 \mathrm{e} 158$.

[41] A. Allahbakhsh, S. Mazinani, M.R. Kalaee, F. Sharif, Cure kinetics and chemorheology of EPDM/grapheme oxide nanocomposites, Thermochim. Acta 563 (2013) 22e32.

[42] L. Valentini, M. Cardinali, J.M. Kenny, M. Prato, O. Monticelli, A photoresponsive hybrid nanomaterial based on graphene and polyhedral oligomeric silsesquioxanes, Eur. J. Inorg. Chem. (2012) 5282e5287. 